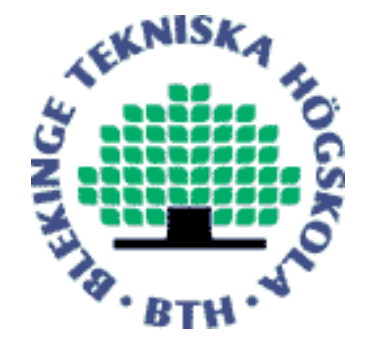

Copyright (C) 2013 IEEE.

Citation for the published paper:

Ground Moving Target Detection and Estimation with Different Sar Linear Flight Tracks

Viet Thuy Vu, Thomas Sjögren, Mats Pettersson

International Geoscience and Remote Sensing Symposium

2013 Melbourne

This material is posted here with permission of the IEEE. Such permission of the IEEE does not in any way imply IEEE endorsement of any of BTH's products or services Internal or personal use of this material is permitted. However, permission to reprint/republish this material for advertising or promotional purposes or for creating new collective works for resale or redistribution must be obtained from the IEEE by sending a blank email message to pubs-permissions@iee.org.

By choosing to view this document, you agree to all provisions of the copyright laws protecting it. 


\title{
GROUND MOVING TARGET DETECTION AND ESTIMATION WITH DIFFERENT SAR LINEAR FLIGHT TRACKS
}

\author{
Viet T. Vu, Thomas K. Sjögren, Mats I. Pettersson* \\ Blekinge Institute of Technology, Campus Gräsvik, 37179 Karlskrona, Sweden
}

\begin{abstract}
The paper proposes a ground moving target detection and estimation method aiming at Ultra Wide Band and -Beam (UWB) Synthetic Aperture Radar (SAR) systems. The method is developed on the moving target detection by focusing technique and requires a SAR system flying with two different linear flight track. The method allows us to detect ground moving target, even hidden by clutter, and to estimate the target parameters such as speed and direction of motion. The accuracy of the estimations depends strongly on the computational cost and can therefore be controlled. The proposal is tested with the simulated CARABAS-II data.
\end{abstract}

Index Terms - UWB, SAR, detection, estimation, NRS

\section{INTRODUCTION}

Ground Moving Target Indication (GMTI) is one of the most important applications of Synthetic Aperture Radar (SAR). SAR GMTI does not only allows us to detect moving targets in an observed area but also helps us to get the images of those targets. Such information is very important for both surveillance and reconnaissance. The GMTI methods developed for SAR are usually based on the effects caused by motion such as Doppler, shadow, phase error and so forth. For multichannel SAR systems, GMTI can be relied on space time processing techniques such as Displaced Phase Center Antenna (DPCA) and Space Time Adaptive Processing (STAP).

Ultra Wide Band and -Beam (UWB) SAR systems which indicates SAR systems utilizing large fractional bandwidth signal and wide beamwidth antenna have emerged and exist besides SAR systems with traditional small fractional bandwdith and narrow beamwidth antenna. Examples of such systems can be found with CARABAS-II operating in the lower VHF-band from 20 to $90 \mathrm{MHz}$ [1], LORA in the VHF- and UHF-bands from 200 to $800 \mathrm{MHz}$ [2], and P-3 with a bandwidth of $515 \mathrm{MHz}$ in the VHF/UHF-bands [3]. These systems enable high resolution imaging thanks to the relatively

\footnotetext{
*The authors would like to thank the KK-Foundation for the financial support in this research project, the Swedish Defence Research Agency, Saab Bofors Dynamics, Saab Electronic Defence Systems and RUAG Space for their cooperation.
}

large bandwidth and possibly wide synthetic angle. Especially, the systems operating at low radar frequencies, e.g. CARABAS-II, allows foliage penetration which can even detect changes in dense forested areas or under camouflage. The GMTI methods developed for UWB SAR cannot only be based on the common effects caused by motion but also on the characteristics of UWB.

The moving target detection by focusing technique is one of the GMTI methods with the help of UWB characteristics [4]. The long integration time associated with UWB SAR strongly disperses the energy reflected from moving targets in a ground scene to different range bin. If we synthesize a large aperture from different aperture positions, for example using Global Backprojection (GBP) [5], the energy reflected from moving targets spread into a large amount of grids. Specifically, in a SAR image, moving targets smear as either hyperbolic or elliptic curves and are obscured by clutter. The moving target detection by focusing technique using Normalized Relative Speed (NRS) to process the data so that the moving targets are refocused to their original shape in the SAR image and at the same time the clutter is strongly defocused or in other words strongly suppressed. The technique allows us to detect moving targets, even hidden by clutter like foliage, using single channel data and gives us directly an initial estimation for those moving target, i.e. NRS. Further information about the detected moving targets such as speed and direction of motion are not possible with the current technique. In addition, the technique also faces challenges in the certain cases, e.g. when the target moves to the blind direction [6] or in in an environment with strong clutter. In such the cases, the detection technique needs to combine with the space time processing techniques for initial clutter suppression.

The objective of this paper is to extend the moving target detection by focusing technique to a detection and estimation method aiming at UWB SAR systems. The method is then validated with simulated data. The simulations are based on the parameters of CARABAS-II, a Swedish UWB SAR system.

The paper is organized as follows. Section 2 extends the moving target detection by focusing technique for a more general case. The approach for moving target estimation is introduced in section 3. The simulation results are presented in section 4 . Section 5 provides the conclusions. 


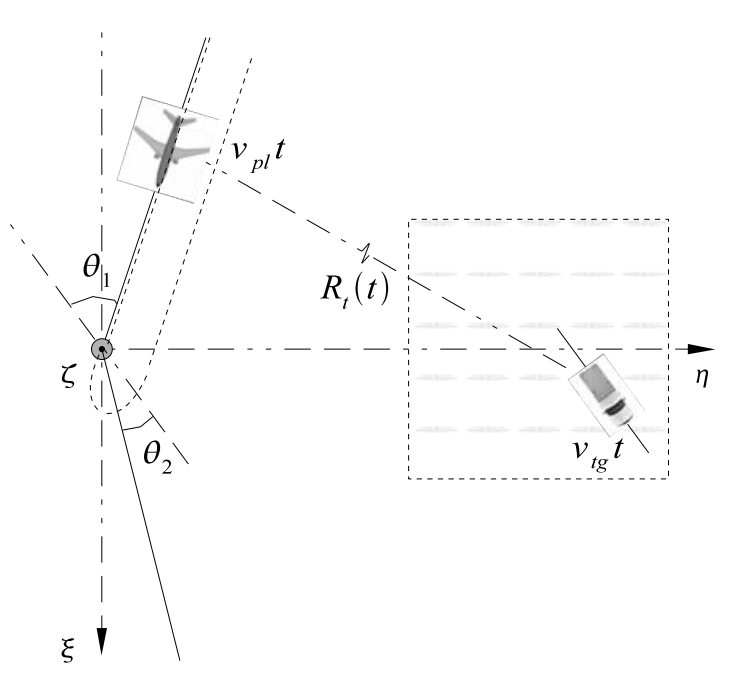

Fig. 1. SAR geometry.

\section{MOVING TARGET DETECTION BY FOCUSING}

Let's consider a SAR system whose dual flight tracks are symmetrical with respect to the $\eta$ axis as shown in Fig. 1 . The movement of the platform is assumed to be linear and with constant speeds, i.e. no acceleration. Without losing the generality, the movement of the platform along the first flight track can be expressed by

$$
\begin{aligned}
& \xi_{p l}(t)=v_{p l, \xi} t-\xi_{p l, 0} \\
& \eta_{p l}(t)=-v_{p l, \eta} t+\eta_{p l, 0} \quad \text { for } \quad 0 \leq t \leq t_{0} / 2 \\
& \zeta_{p l}(t)=\zeta_{p l, 0}
\end{aligned}
$$

where $v_{p l, \xi}$ and $v_{p l, \eta}$ are the speed components and therefore $\sqrt{v_{p l, \xi}^{2}+v_{p l, \eta}^{2}}=v_{p l}$. The initial coordinates of the platform in a Cartesian coordinate system are given by $\left(\xi_{0}, \eta_{0}, \zeta_{0}\right)$. The integration time is denoted by $t_{0}$. The initial coordinates and the integration time are connected together by $\xi_{p l, 0}=$ $v_{p l, \xi} t_{0} / 2$ and $\eta_{p l, 0}=v_{p l, \eta} t_{0} / 2$. The SAR system is assumed to illuminate a ground scene where there is a moving target, e.g. a truck. The movement of the target on the ground is also assumed to be linear and with constant speeds. The mathematical representation for the movement of the target on the ground can be found by

$$
\begin{aligned}
& \xi_{t g}(t)=v_{t g, \xi} t+\xi_{t g, 0} \\
& \eta_{t g}(t)=v_{t g, \eta} t+\eta_{t g, 0} \quad \text { for } \quad 0 \leq t \leq t_{0} \\
& \zeta_{t g}(t)=0
\end{aligned}
$$

where $v_{t g, \xi}$ and $v_{t g, \eta}$ are the speed components and we also have $\sqrt{v_{t g, \xi}^{2}+v_{t g, \eta}^{2}}=v_{t g}$. Based on (1) and (2), the true range calculated for the SAR aperture positions along the first flight track and the ground moving target is given by

$$
\begin{aligned}
R_{t}(t) & =\left\{\left(v_{p l, \xi} t-\xi_{p l, 0}-v_{t g, \xi} t-\xi_{t g, 0}\right)^{2}\right. \\
& \left.=+\left(-v_{p l, \eta} t+\eta_{p l, 0}-v_{t g, \eta} t-\eta_{t g, 0}\right)^{2}+\zeta_{p l, 0}^{2}\right\}^{\frac{1}{2}}
\end{aligned}
$$

If the true range history is used to reconstruct the SAR scene, the ground moving target in the SAR scene will be displaced and smeared in the SAR image in the form of either hyperbolic or elliptic curve. According to the concept of the relative speed between two objects in motion, one moving object is seen to be stationary for the other if it is assumed to move with the relative speed between them. In other words, a ground moving target can be seen to be stationary for a SAR platform if the SAR platform is assumed to move with the relative speed between them. If we call $\gamma_{1}$ is the normalized relative speed between the SAR platform moving along the first flight track and the ground moving target, the relative speed of the SAR platform will be $\gamma_{1} v_{p l}$. The ground moving target is seen to be stationary at certain coordinates $\left(\xi_{1}, \eta_{1}\right)$ by the SAR platform and the range history is estimated by

$$
\begin{aligned}
R_{p}(t) & =\left\{\left(\gamma_{1} v_{p l, \xi} t-\xi_{p l, 0}-\xi_{1}\right)^{2}\right. \\
& \left.=+\left(-\gamma_{1} v_{p l, \eta} t+\eta_{p l, 0}-\eta_{1}\right)^{2}+\zeta_{p l, 0}^{2}\right\}^{\frac{1}{2}}
\end{aligned}
$$

In this case, the following circumstance must be fulfilled during the integration time

$$
R_{t}(t)=R_{p}(t) \text { for } 0 \leq t \leq t_{0} / 2
$$

Equation (5) will have the form of $\sqrt{a}=\sqrt{b}$ if we insert (4) and (5) in (5). To solve such equation in order to find $\gamma_{1}$, we first take the square both sides of the equation. This result in

$$
\begin{aligned}
& \left(v_{p l, \xi} t-\xi_{p l, 0}-v_{t g, \xi} t-\xi_{t g, 0}\right)^{2} \\
& \quad+\left(-v_{p l, \eta} t+\eta_{p l, 0}-v_{t g, \eta} t-\eta_{t g, 0}\right)^{2} \\
& \quad=\left(\gamma_{1} v_{p l, \xi} t-\xi_{p l, 0}-\xi_{1}\right)^{2}+\left(-\gamma_{1} v_{p l, \eta} t+\eta_{p l, 0}-\eta_{1}\right)^{2}
\end{aligned}
$$

Equation (7) is a second order equation and has the form $a t^{2}+$ $b t+c=0$. However, to be assure that the condition (5) satisfies at any time $t$ during the integration time, the set of equations (7), which corresponds to the terms $t^{0}, t^{1}$ and $t^{2}$, is required to be fulfilled. The last equation in (7) results in NRS

$$
\gamma_{1}=\frac{\sqrt{\left(v_{p l, \xi}-v_{t g, \xi}\right)^{2}+\left(v_{p l, \eta}+v_{t g, \eta}\right)^{2}}}{v_{p l}}
$$

We can also show that (8) can be rewitten by

$$
\gamma_{1}=\frac{\sqrt{v_{p l}^{2}-2 v_{p l} v_{t g} \cos \theta_{1}+v_{t g}^{2}}}{v_{p l}}
$$




$$
\left\{\begin{array}{l}
\left(\xi_{p l, 0}+\xi_{t g, 0}\right)^{2}+\left(\eta_{p l, 0}-\eta_{t g, 0}\right)^{2}-\left(\xi_{p l, 0}+\xi_{1}\right)^{2}-\left(\eta_{p l, 0}-\eta_{1}\right)^{2}=0 \\
\left(v_{p l, \xi}-v_{t g, \xi}\right)\left(\xi_{p l, 0}+\xi_{t g, 0}\right)+\left(v_{p l, \eta}+v_{t g, \eta}\right)\left(\eta_{p l, 0}-\eta_{t g, 0}\right)-\gamma_{1}\left[v_{p l, \xi}\left(\xi_{p l, 0}+\xi_{1}\right)+v_{p l, \eta}\left(\eta_{p l, 0}-\eta_{1}\right)\right]=0 \\
\left(v_{p l, \xi}-v_{t g, \xi}\right)^{2}+\left(v_{p l, \eta}+v_{t g, \eta}\right)^{2}-\gamma_{1}^{2}\left(v_{p l, \xi}^{2}+v_{p l, \eta}^{2}\right)=0
\end{array}\right.
$$

where $\theta_{1}$ is the angle formed by the first flight track and the target trajectory as shown in Fig. 1. The first two equations in (7) give the coordinates $\left(\xi_{1}, \eta_{1}\right)$ which are not discussed in detail in this paper.

For detection, different hypotheses of NRS $\gamma_{p}$ needs to be tested. The step size between the hypotheses and the range of hypotheses are presented in detail in [4]. At a hypothesis $\gamma_{p} \approx \gamma_{1}$, the moving target is strongly focused in the reconstructed SAR scene and at the same time the clutter is strongly smeared. If $\Lambda$ is a detection threshold and if the intensity of the focused moving target is larger than the threshold while the intensity of smeared clutter, it will be possible to detect the moving target.

However, we can easily see that if the target moves in the so-called blind direction [6], i.e.

$$
\theta_{1}=\operatorname{Arccos}\left(\frac{v_{t g}}{2 v_{p l}}\right)
$$

we cannot distinguish between the moving target and the clutter as the result of $\gamma_{1}=1$. This will be one of the technical issues will be solved in the redetection stage presented in the next section.

\section{REDETECTION AND ESTIMATION}

Let's consider the second flight track of the SAR system. Similarly, the movement of the platform along the second flight track can be shown to be

$$
\begin{aligned}
& \xi_{p l}(t)=v_{p l, \xi} t-\xi_{p l, 0} \\
& \eta_{p l}(t)=v_{p l, \eta} t-\eta_{p l, 0} \quad \text { for } \quad t_{0} / 2 \leq t \leq 2_{0} \\
& \zeta_{p l}(t)=\zeta_{p l, 0}
\end{aligned}
$$

We can find NRS and the corresponding coordinates $\left(\xi_{2}, \eta_{2}\right)$ in the similar way presented in section 2 . The mathematical representation is given by

$$
\gamma_{2}=\frac{\sqrt{v_{p l}^{2}-2 v_{p l} v_{t g} \cos \theta_{2}+v_{t g}^{2}}}{v_{p l}}
$$

where $\theta_{2}$ is the angle formed by the second flight track and the target trajectory. The relationship between $\theta_{1}$ and $\theta_{2}$ can be found from Fig. 1 as

$$
\theta_{2}=\theta_{p}-\left(\pi-\theta_{1}\right)
$$

where $\theta_{p}$ is the angle formed by the first and the second flight tracks. In principle, we can find the presentations for $v_{t g}$ and $\theta_{1}$ or $\theta_{2}$ based on (9), (12) and (13). However, the mathematical presentations are quite complicated and can only be simplified in some special cases, e.g. the first and second flight tracks are perpendicular or parallel, i.e. $\theta_{p}=\pi / 2$ or $\theta_{p}=2 \pi$. In this paper, we present the solutions only for the special case where $\theta_{p}=2 \pi$. This means the first and second flight tracks are identical. The difference is only the direction of motion, i.e. opposite. In this special case, (12) can be rewritten by

$$
\gamma_{2}=\frac{\sqrt{v_{p l}^{2}+2 v_{p l} v_{t g} \cos \theta_{1}+v_{t g}^{2}}}{v_{p l}}
$$

Even if the target moves in the blind direction for the first flight track and causes $\gamma_{1}=1$, that direction of motion is not the blind direction for the first flight track and therefore $\gamma_{2} \neq 1$.

The relationship between $\gamma_{1}$ and $\gamma_{2}$ can be found from (8) and (14)

$$
\gamma_{2}=\frac{\sqrt{\left(2-\gamma_{1}^{2}\right) v_{p l}^{2}+2 v_{t g}^{2}}}{v_{p l}}
$$

Since the speed of the ground moving target is much smaller than the speed of the SAR platform, i.e. $v_{t g} \ll v_{p l}$, (15) can be approximated by

$$
\gamma_{2} \approx \sqrt{2-\gamma_{1}^{2}}+\left(\frac{v_{t g}}{v_{p l}}\right)^{2}
$$

The approximated relationship (16) allows us to limit the range of hypotheses $\gamma_{p}$ in the redection stage. We can easily find the presentations of $v_{t g}, \theta_{1}$ and $\theta_{2}$ in this special case as

$$
v_{t g}=v_{p l} \sqrt{\frac{\left(\gamma_{1}^{2}+\gamma_{2}^{2}-2\right)}{2}}
$$

and

$$
\theta_{1}=\theta_{2}-\pi=\operatorname{Arccos}\left(\frac{\gamma_{2}^{2}-\gamma_{1}^{2}}{2 \sqrt{2\left(\gamma_{1}^{2}+\gamma_{2}^{2}-2\right)}}\right)
$$

The special case, where $\theta_{p}=2 \pi$, will be considered to illustrate the moving target detection and estimation in the next section.

\section{PROPOSAL VALIDITY}

In this section, we present some simulation results to verify the proposal of moving target detection and estimation by focusing. In the simulations, we use a geometry similar to the 
Table 1. Parameters of the simulated platform.

\begin{tabular}{|c||c|}
\hline Parameter & Value \\
\hline The highest frequency processed & $82 \mathrm{MHz}$ \\
The lowest frequency processed & $22 \mathrm{MHz}$ \\
Platform speed $v_{p l}$ & $126 \mathrm{~m} / \mathrm{s}$ \\
Flight altitude & $3700 \mathrm{~m}$ \\
Minimum range to the aim point & $7150 \mathrm{~m}$ \\
Pulse Repetition Frequency (PRF) & 137 \\
Aperture step & $0.9375 \mathrm{~m}$ \\
\hline
\end{tabular}

one given in Fig. 1. The first flight track is considered for detection and the same flight track but in the opposite direction is considered for redetection and estimation.

\subsection{Arrangement}

The simulated SAR system is based only on CARABAS-II's parameters in reality [2]. The main parameters of this system are summarized in Table 1. In the simulation, the number of aperture positions is selected by about 3600 corresponding to a synthetic aperture of $30^{\circ}$ with respect to the center of the simulated ground scene. The angle formed by the first flight track and the $\xi$ axis is $25^{\circ}$.

The simulated ground scene consists of a moving target which is the subject to be detected and estimated and clutter covering the moving target. The moving target is a point-like scatterer and its Radar Cross Section (RCS) is normalized to 1. The speed of the target is chosen by $10 \mathrm{~m} / \mathrm{s}$ and the direction of motion is $15^{\circ}$ with respect to the $\xi$ axis. The clutter is a set of point-like scatterers and equally spaced in the ground scene. The RCS of the clutter are given by the pseudorandom values drawn from the standard uniform distribution on the open interval $(0,1)$ and scaled with a factor of $1 / 2$.

Fig. 2 shows the SAR image of the simulated ground scene with the GBP algorithm using the true ranges calculated by (4). The image is reconstructed with the data collected during the SAR platform moving along the first flight track. The area of the reconstructed SAR scene is $512 \mathrm{~m} \times 512 \mathrm{~m}$, and the sampling azimuth and range are $1 \mathrm{~m} \times 1 \mathrm{~m}$. As shown in Fig. 2, the clutter is well focused and appears everywhere. At the same time, the moving target is blurred in the SAR image and totally obscured by the clutter.

\subsection{Detection}

An optimum quantization step size between hypotheses is selected by $\Delta \gamma=5 \cdot 10^{-3}$. This selection corresponds to the threshold of $h_{\text {lim }}=3 \mathrm{~dB}$ allowing maximum loss of $3 \mathrm{~dB}$ from the peak intensity of the moving target in the SAR image. If we need to detect moving targets whose speed is less

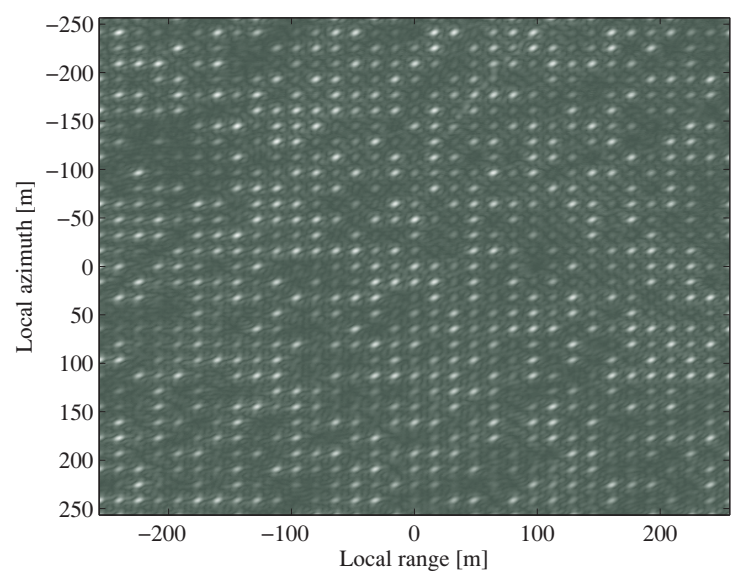

Fig. 2. SAR image of the simulated ground scene. The image is reconstructed with the first flight track data.

than $10 \%$ of the platform speed, the range of hypotheses is only in the interval $\gamma_{p} \in[0.9,1.1]$. We also assume here that the area of interest or the observed area is whole SAR scene. In reality, areas of interest are also usually very large, e.g. forest where moving targets can be hidden by thick foliage, a water area where vessels are prevented to go into, or a long road where over speed vehicles need to be indicated.

Fig. 3 shows the relationship between $\gamma_{p}$ and the retrieved peak intensities in the area of interest (continuous curve) or in other words the detection result. As observed the 2-D graph, two peak intensities occur at the hypotheses $\gamma_{p}=1$ and $\gamma_{p}=$ 0.940. The former belongs to the clutter and the other the moving target which need to be detected. If we set a detection threshold of, for example $\Lambda=3 \mathrm{~dB}$ and apply it for the found peak intensities, the presence of a moving with NRS of 0.940 in the area of interest will be indicated. Hence, the moving target is detected and the estimated NRS for the target is about $\breve{\gamma}_{1}=0.940$.

\subsection{Redetection}

With the value of $\gamma_{1}$ retrieved in the detection stage, we can sparsely estimate $\gamma_{2}$ using (16). If we use the first term of (16) to estimate $\gamma_{2}$, the maximum error caused by this estimation will be given by the second term of (16). Hence, the first term gives $\gamma_{2} \approx 1.055$ while the second term helps us to define the range of hypotheses for the redetection stage $\gamma_{p} \in$ $[1.045,1.065]$. It is obvious that this range of hypotheses is much narrower than the one for the detection stage (more than 8 times). For redetection, we use the same step size as in the detection stage, i.e. $\Delta \gamma=5 \cdot 10^{-3}$. Based on a similar 2-D graph of the relationship between $\gamma_{p}$ and the peak intensities also shown in Fig. 2 (dashed curve), we can redetect the same moving target and retrieve $\breve{\gamma}_{2}=1.06$. 